\section{APLICACIÓN DE LA TEORÍA Y MÉTODO DE ANÁLISIS DEL ARTE PREHISTÓRICO DE C. ALONSO DEL REAL A AUGA DA LAXE, GONDOMAR, PONTEVEDRA, ROCA CON GRABADOS DE LA EDAD DEL BRONCE EN GALICIA}

\author{
APPLICATION OF THE PREHISTORIC \\ ART THEORY AND METHOD OF \\ ANALYSIS OF C. ALONSO DEL REAL TO \\ AUGA DA LAXE, GONDOMAR, \\ PONTEVEDRA, ROCK WITH BRONZE \\ AGE ENGRAVINGS IN GALICIA
}

JOSÉ MANUEL VÁZQUEZ VARELA (*)

\section{RESUMEN}

Se aplican las concepciones epistemológicas y metodológicas del profesor C. Alonso del Real (1914-1993) al estudio de Auga da Laxe I, yacimiento de arte rupestre de la Edad del Bronce en el Noroeste de la Península Ibérica. Su enfoque holístico resulta eficaz y aporta nuevos datos sobre la función y el significado de los grabados de armas de la Edad del Bronce. Auga da Laxe puede ser un lugar de celebración de rituales de agregación de guerreros.

\begin{abstract}
The epistemology and methodology concepts of professor C. Alonso del Real (1914-1993) are applied to the study of Auga da Laxe I, rock with Bronze Age engra-
\end{abstract}

(*) Departamento de Historia 1. Facultade de Xeografía e Historia. Universidade de Santiago de Compostela. 15703 Santiago.

$\mathrm{El}$ artículo fue remitido en su versión final el 13-IV-94. vings in North-west Spain. The holistic perspective provide new data about the function and meaning of engravings of arms in the Bronze Age. Auga da Laxe seems to be a place for holding warrior aggregation rituals.

Palabras clave: Epistemología. Metodología, Petroglifos. Edad del Bronce.

Key words: Epistemology. Methodology. Rock carvings. Bronze Age.

\section{INTRODUCCIÓN}

El profesor Carlos Alonso del Real (19141993) en su contribución al homenaje a H. Breuil publicado en Barcelona (Alonso, 1964) dice que una buena forma de honrar a un maestro es contrastar y matizar su pensamiento con la aportación de nuevos logros. Este trabajo se enmarca dentro de esta línea con la doble pre- 
tensein de exponer los aspectos fundamentales del pensamiento y del metodo del profesory hacer una aplicacion del mismo con algunos matices a un caro concreto: el análisis de una roca con grabados de la Edad del Bronce en Galicia

El estudio del arte rupestre fue uno de sus preferidos entre otros motivos porque lo consideraba un documento privilegiado para conocer aspectos importantes del pasado entre ellos los de iipo social y simbólico.

En sus trabajos. en los que sigue las lineas de H. Breuil. A. Laming Emperaire y A. LeroiGourhan, con respeto y autonomía. adopta los planteamientos de Panofsky (Alonso. 1975) y recurre a paralelos etnológicos, etnohistóricos. folklóricos, arqueológicos e históricos para la interpretación de la obra de arte.

C. Alonso del Real concibe la Prehistoria como un período histórico en el que, a través del estudio arqueológico, de la visión interdisciplinar y holística se puede analizar el testimonio artístico como un segmento de la cultura a la que pertenece a la luz de la cual ha de entenderse y sobre algunos de cuyos aspectos ofrece información (Alonso, 1974).

En su búsqueda del mayor conocimiento posible sobre el pasado, en especial en los aspectos ideológicos, religiosos y sociales, recurre al empleo de modelos y métodos de diferentes escuelas a veces aparentemente contradictorias tales como la fenomenología de Mircea Eliade, el marxismo o la escuela histórico-cultural de Viena (Alonso, 1961, 1974).

Si bien emplea criterios de A. Leroi-Gourhan, usa teoría etnológicas aparentemente incompatibles con los principios de éste. Su formación como humanista y su inquietud personal, para la cual nada humano era extraño, le lleva a emplear una amplia variedad de paralelos históricos y etnológicos en sus análisis, en los que trata de conocer numerosos aspectos del pasado a menudo poco tratados. Esta labor, en la que se refleja el conocimiento de un gran número de culturas desde la América precolombina hasta Melanesia, se ve facilitada por sus dotes de políglota que le permiten manejar estudios y documentos originales en diversas lenguas desde el arameo hasta el ruso pasando por el quechua.

Como muestra de sus concepciones epistemológicas y metodológicas se ofrece de forma breve un ejemplo de su aplicación a la esta- cion arqueológica conocida como Auga da I.axe I. V'incios. en el ayuntamiento de Gondomar. en el suroeste de la provincia de Pontevedra (Fig. 1).

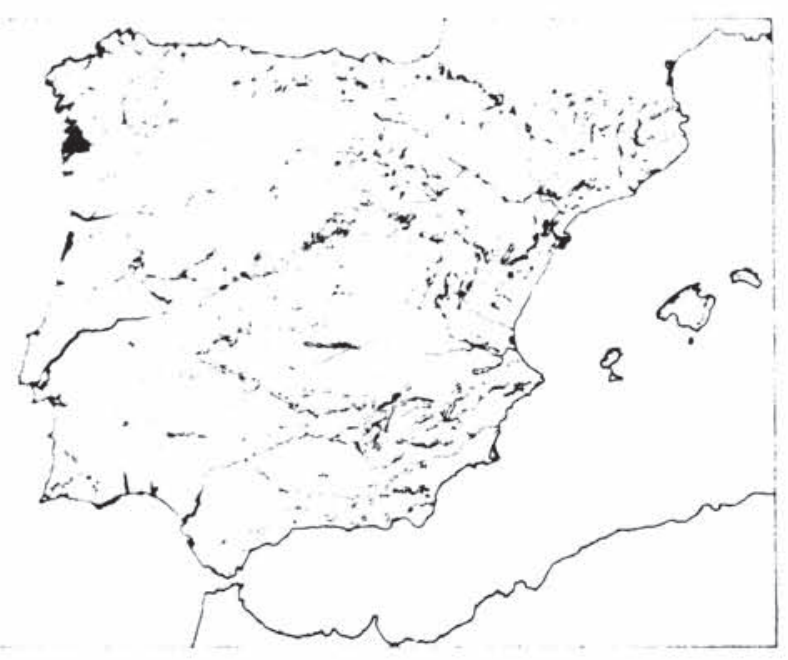

Fig. 1. Situación del yacimiento de Auga da Laxe I, en el Suroeste de la provincia de Pontevedra

\section{EL YACIMIENTO}

El yacimiento de Auga da Laxe I es una gran roca granítica del Monte dos Arruidos en la falda occidental de la sierra do Galiñeiro, donde afloran numerosas rocas de buen tamaño en algunas de las cuales se han localizado diferentes tipos de grabados. Es el caso de las tres denominadas Auga da Laxe II, III y IV con representaciones de armas situadas en un radio de menos de doscientos metros en torno a esta estación (Costas et alii, 1984). La roca, de grano grueso, presenta en su parte superior varias pilas, en las que no se aprecia intervención humana, que retienen el agua de la lluvia.

Los grabados se presentan en la cara oriental de pendiente bastante pronunciada y forman un total de veintiséis compuesto por diez puñales o espadas cortas, una larga, seis alabardas, ocho escutiformes y algunos trazos cuya lectura no resulta posible debido a la erosión. Estas armas están colocadas verticalmente en el sentido de la pendiente principal de la cara de la roca. Da la impresión de que si no fueron hechas por una misma mano sí lo fueron respetando un canon, por la uniformidad del tamaño y de su posición a lo largo del conjunto (Fig. 2). 


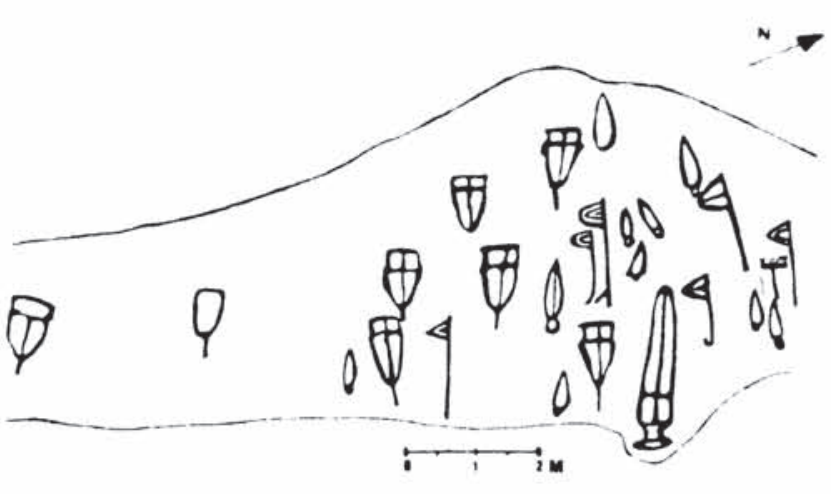

Fig. 2. Calco de los grabados de Auga da Laxe I. Modificado por N. Calo a partir de Costas et alii (1984)

El yacimiento ha sido objeto de varias publicaciones y referencias (Costas et alii, 1984; Vázquez, 1990, 1993) y es uno de los más llamativos de la región por el número de grabados, el tamaño de la roca y la impresión de conjunto realizado intencionalmente en un corto espacio de tiempo al comienzo de la Edad del Bronce, si bien cabe la posibilidad de que la gran espada sea posterior al resto de las figuras.

\section{ANÁLISIS ARQUEOLÓGICO}

El análisis de los grabados prehistóricos al aire libre en Galicia se ha mostrado útil para la reconstrucción de aspectos de la sociedad de la primera parte de la Edad del Bronce. La comparación de los objetos representados con los restantes componentes del registro arqueológico permite establecer hipótesis interpretativas sobre aspectos de la organización social y la ideología especialmente en lo relativo a la jerarquización de las comunidades y a la importancia de lo bélico (Vázquez, 1990, 1991, 1992).

Los grabados de armas tienden a aparecer disociados de los restantes y son los más figurativos. En algunos puñales se puede apreciar la empuñadura separada de la hoja y su detallado bisel. En varias alabardas se aprecia el biselado y el nervio central de la hoja.

Suelen formar composiciones que a menudo presentan un claro plan de organización en el que las armas se concentran en un espacio delimitado, orientadas en la misma dirección o bien en grupos afrontados.
Son unos de los pocos temas que se representan en la mavoria de las ocasiones a una escala igual o ligeramente superior a la natural. En algún caso su tamaño es muy superior a la figura humana que la porta como ocurre en $\mathbf{A}$ Pedra das Ferraduras de Fentans. Cotobade. Pontevedra (Vázquez. 199.3).

Desde el punto de vista espacial se distinguen dos tipos de estaciones: uno compuesto por un reducido número de figuras que se encuentran en una roca poco visible. El otro lo integran aquellas que agrupan un conjunto mayor de armas en una roca de dimensiones más grandes y más visible en el paisaje: a menudo se hallan en un lugar en el que se puede asentar un colectivo humano numeroso con comodidad dando la impresión de que en algún momento servían de escenario a alguna actividad.

Estos rasgos que diferencian a las armas del conjunto de los grabados se pueden interpretar como modos de marcar su importancia y carácter peculiar.

En el registro arqueológico los ejemplares de armas aparecen en hallazgos descontextualizados, en cistas funerarias, en túmulos, en el interior de la cámara de un monumento megalítico de corredor, en la parte superior del túmulo de otro y en depósitos. Atendiendo a la distribución específica para cada tipo para la provincia de Pontevedra recientemente estudiada (Comendador, 1992) se puede señalar que las nueve hachas planas aparecieron sin contexto claro, si bien una de ellas se encontraba en una grieta de una roca donde hay grabados; de las seis puntas Palmela, tres proceden de túmulos y tres no tienen referencia arqueológica clara; de los tres puñales de espigo uno procede de un túmulo y dos de una misma cista, la de Atios.

El análisis de la frecuencia de aparición de los diferentes tipos revela que en Galicia hay más de veintitrés hachas, más de diecinueve puntas Palmela, más de diecisiete puñales de espigo y una alabarda segura, la de Leiro, Rianxo, A Coruña y otra problemática en el depósito de Roufeiro, Sarreaus da Limia, Ourense (Meijide, 1991).

El contraste entre los objetos reales y sus representaciones viene marcado por la ausencia de grabados de hachas, de puntas Palmela y la abundancia de puñales en relación con las alabardas aunque en una proporción relativamente equilibrada muy lejos de la relación existente entre los objetos reales donde la relación sería 
de diecisiete a uno o decisiete a dos. De hecho en algunas estaciones rupestres de cierto tamaño hay un numero muy parecido de ambos tipos. En estos datos se aprecia la selección que efectúa el grabador quien destaca algunos de los objetos y lo hace en unas proporciones muy diferentes a las de la realidad señalando de esta manera su importancia simbólica.

Los ajuares contenidos en las cistas caracteristicas del comienzo de la Edad del Bronce proporcionan datos que apoyan la existencia de una jerarquización social en la que el aspecto bélico tenia un papel de cierta importancia.

En una sola tumba, la de Atios. O Porriño. Pontevedra, aparecen dos punales, uno corto y otro largo, que a su vez están acompañados por dos cilindros de oro y dos joyas de plata. Sólo en otra cista de la necrópolis de A Pedrosa en el sur de Ourense aparece una espiral de plata. En Taraio, A Coruña se encontró un puñal y un vasito cerámico, en Fariñas un puñal largo, en Carnota un puñal y un brazal de arquero, en la de As Antas un par de vasos cerámicos y puñal. La máxima diferencia va de la cista de Budiño a una de Gandón donde se encuentran restos de un niño sin ajuar. Estos datos apoyan la idea de la importancia de lo bélico y que la jerarquía social debía de estar señalada, como en la tumba de Budiño, por la posesión de joyas y armas (Vázquez, 1994). Soluciones semejantes se encuentran en esta época en numerosas comunidades europeas en las que se comprueba por varios datos arqueológicos la fiabilidad de esta interpretación.

\section{FUNCIÓN Y SIGNIFICADO}

\section{Estado de la cuestión}

En trabajos anteriores (Vázquez, 1991, 1992) se interpretó la presencia de grabados de armas y de las escasas escenas en las que aparece la figura humana como testimonio de una sociedad donde se proclama para legitimarla la idea de la importancia del varón, de sus actividades como la guerra, la caza y quizás el pastoreo y la de las armas y los guerreros.

Los temas representados reflejan los valores del varón y del guerrero propios de una sociedad orientada en cuanto al poder en torno al hombre, quizás de tipo patrilineal y patriarcal, como se documenta en otras culturas europeas de la Edad del Bronce.
Las representaciones de armas se han intepretado desde el punto de vista religioso como un posible testimonio de la hoplolatria. Aquéllas podrian ser símbolos de los guerreros que las consagran a una figura religiosa vinculada con la guerra o bien representaciones de ésta de acuerdo con paralelos arqueológicos. etnológicos e históricos (Vázquez, 1994).

La posibilidad que aqui se desarrolla es la de considerar que Auga da Laxe y los yacimientos con grabados de armas del mismo tipo pueden ser lugares de celebración de rituales de agregacion de guerreros.

\section{La aportación de la etología humana}

El análisis de los grabados desde la perspectiva de la etología humana, a pesar del recelo con que suele ser recibida por parte de algunos humanistas, también resulta provechoso, al menos, para generar hipótesis interpretativas a contrastar por otras ciencias sociales. Si Auga da Laxe ha sido realizada de una vez o en un período de tiempo muy corto puede representar un conjunto de guerreros en un rito de afirmación agresivo, con las armas hacia arriba, en posición de mensaje coercitivo de amenaza que al tiempo recuerda rituales de saludo al extraño que llega a un territorio ajeno. Encierra un mensaje doble, ambiguo, en el que se exhiben señales de amistad al tiempo que de afirmación del poderío del grupo relacionadas con la afirmación sobre el propio territorio (Eib-Eibesfeldt. 1993). En este sentido el lector puede recordar las escenas de encuentro entre viajeros occidentales y grupos de otras culturas en la bibliografía y la filmografía, sobre todo, del género de aventuras y viajes en las que las poblaciones etnográficas salen armadas en un doble intento de acercamiento e intimidación.

Desde esta perspectiva, Auga da Laxe podría estar marcando el límite del territorio de una comunidad o de un grupo concreto lo cual se ve apoyado por su proximidad a las vías de comunicación tradicionales de la zona.

\section{Los grupos de guerreros}

Las recientes investigaciones sobre los pueblos prerromanos del Noroeste de la Península han descubierto la existencia de grupos o cofradías de guerreros que encajan muy bien con las propias del mundo céltico continental e insular 
y con los pueblos indoeuropeos en general (García, 1991).

Son grupos de guerreros autónomos o integrados en la tribu; que tienen un carácter marginal; practican la caza y la guerra, que suele ser de pillaje y de ostentación para adquirir estatus: están vinculados con una divinidad guerrera a la que ofrecen sacrificios, a veces en lugares sagrados específicos como grandes rocas en zonas marginales, en la tierra de nadie, en los límites de comunidades donde se celebran asambleas, fiestas religiosas, ritos iniciáticos y a veces reuniones sociales, administrativas, jurídicas y económicas.

Hay grupos que viven gran parte del año en los lugares marginales donde hacen la guerra, cazan y celebran ritos iniciáticos y otros, y a veces se integran en los poblados bajo la autoridad superior.

Algunos colectivos tienen carácter marginal y antisocial. Así, por ejemplo, en la literatura irlandesa Fión recibe una educación marginal e iniciática, vive al aire libre en la montaña donde caza y lleva un tipo de vida en la que lo bélico se une con lo heroico y lo mágico (García, 1991).

Si bien estos rasgos de guerrero se detectan durante la Edad del Hierro en Europa Occidental y bastantes de ellos son comunes a las comunidades de guerreros indoeuropeos, no se puede extrapolar la existencia de este conjunto de rasgos al comienzo de la Edad del Bronce, aunque sí es posible usarlos como hipótesis de trabajo para analizar las rocas con grabados de armas y ver hasta qué punto pueden reflejarse en ellas algunas de las características citadas.

Auga da Laxe muestra un conjunto de atributos que encajan bien en el modelo descrito de comportamiento de los guerreros:

1. Roca de buen tamaño con grabados de un conjunto de armas que parecen reflejar, de un modo metafórico, mediante la metonimia de la parte por el todo, una reunión de guerreros.

2. Su posición en el saltus, una zona marginal con relación a las tierras más aptas para la producción de alimentos que desde ella se divisan.

3. La existencia de piletas naturales en las que se acumula el agua de la lluvia que pudieran haber sido utilizadas en los sacrificios.

4. Su posición en un lugar de comunicación, la vía de paso natural del valle a lo más montañoso, marginal e improductivo del territorio.

\section{Zona rica en caza.}

6. Un área de monte que puede servir como límite entre las comunidades que se asientan en el fondo de los valles que se divisan desde allí.

Este conjunto de atributos encaja bien con el escenario y las prácticas de las cofradías de guerreros prerromanos, con el modelo irlandés en el que incluso se graban temas simbólicos en las rocas que constituyen lo esencial de los lugares sagrados (García, 1991) y con el modelo indoeuropeo en general. Por esto se puede afirmar que Auga da Laxe pudo servir de lugar de reunión de grupos de guerreros como los aludidos.

No se entra con ello en la cuestión de la fecha más antigua de la indoeuropeización de las instituciones sociales del territorio, ni por tanto se afirma que tuviesen ese carácter las gentes de la comarca del comienzo de la Edad del Bronce. Además, es posible señalar de acuerdo con los datos etnográficos e históricos que muchas de las características de los guerreros indoeuropeos se dan en sociedades muy distantes en el espacio y el tiempo, como indica Lincoln (1991) al estudiar los paralelos existentes entre los indoiranios y los Nuer de Sudán.

Por ello, aunque los grabados de Auga da Laxe no estuviesen hechos por gentes de cultura indoeuropea, al menos algunas de sus características generales podrían ser coincidentes con las de ellos.

\section{DISCUSIÓN}

La comprobación de esta hipótesis de trabajo requiere un proyecto de investigación actualmente en marcha dedicado al estudio arqueológico del entorno de los petroglifos para detectar los posibles restos de la cultura material que hubiesen quedado después del conjunto de ceremonias realizadas en la zona.

Esta labor de tipo local ha de completarse con otra más amplia para aumentar, a través del registro arqueológico, nuestro conocimiento de los aspectos sociales e ideológicos de la época.

La hipótesis expuesta de Auga da Laxe como lugar de celebración de rituales de agregación a guerreros, parecidos a los de las cofradías de tipo indoeuropeo, se muestra fecunda a la hora de explicar el significado y función del yacimiento ya que, como se ha señalado, si bien es posible su lectura mediante el modelo indo- 
curoper tambien en interpretahle a la lus de las practicas de otras seciedades.

El análisis desde el punto de vista de la etologia humana resulta coincidente con el de los paralelos históricos y etnológicos por lo que las conclusiones derivadas de la aplicación de cada uno de ellos, coincidentes entre sí en lo esencial. se refuerzan y le dan un mayor grado de probabilidad a la interpretación.

\section{CONCLUSIONES}

Los conceptos epistemológicos y metodológicos de C. Alonso del Real. desarrollados y modificados por el autor, resultan útiles para interpretar la función y significado de los petroglifos y conocer aspectos de tipo simbólico, poco asequibles por medio de otros documentos de tipo arqueológico.

Esta aproximación converge en algunos puntos con planteamientos recientes de la arqueología que sigue el modelo anglosajón, lo cual muestra el aspecto moderno del método. en apariencia clásico. de C. Alonso del Real basado en gran parte en corrientes y tendencias distintas.

De ellos resulta la conveniencia de la aplicación de diferentes modelos y métodos en la investigación, y la valoración de la tradición hispánica, entre otras europeas continentales y mediterráneas, a veces injustamente marginadas por aquellos que controlan los circuitos de producción y distribución de ciencia dentro del paradigma dominante.

Algunos círculos académicos que desconocen los logros de otras formas de aproximación al estudio del pasado actúan en la práctica como si ciencia fuese únicamente aquellos que ellos hacen dentro de su reducido universo de escuela con lo cual, al no considerar otros sistemas de estudio, renuncian implícitamente a otro tipo de "verdad" o dicho más elegantemente no pueden disfrutar de los conocimientos de otros colegas.

Por todo ello, el pensamiento de C. Alonso del Real, como el de otros compañeros de su generación, se revela no sólo eficaz, y a pesar del paso del tiempo moderno, sino que sirve de ejemplo para reflexionar sobre la sociología del conocimiento en el campo de nuestra disciplina e invita a la búsqueda de la verdad a través de los varios caminos epistemológicos y metodológicos.

\section{BIBLIOGRAFÍA}

Alons) DH Rtal. C. (1\%61). "Soccologia Pre y Protohistirnca" Instituto de Estudios Politicos. Madrid.

- (19(4): "Notulae Breulianae". En E. Ripol.l. Perello (ed.): Miscelanea en Homenaje al Ahate Henri Breuil (1877.1961). Diput. Prov. Barcelona. Instituto de Prehistoria y Arqueologia. Barcelona. I: 113-121.

- (1974): "El sentido de las pinturas rupestres en las cue. sas de la región ('antábrica". Boletin de la Real Acade. mia de la Historia. CLXXI: 7.76.

- (1975): "Intento de una iconología del arte prehistórico". Actas de las I Jornadas de Metodologia Aplicada de las Ciencias Históricas, I, Prehistoria e Historia An. tigua, Santiago: 17-30.

COMENDAdOR ReY. B. (1993): "Los inicios de la metalurgia: Primeros testimonios de la provincia de Pontevedraw. Brigantium. 7: 185-204.

Costas Goberna, F.: Fernandez Pintos, J.: Goberna Prna. J. y Fernandez Diaz. M. (1984): Petroglifos del litoral sur de la ria de Vigo. Publicaciones del Museo Municipal “Quiñones de León”. 8, Vigo.

Eıв-EIBESFEI.DT, 1. (1993): Biologia del comportamiento humano. Manual de Etología humana. Alianza Editorial. Madrid.

Garcia Fernandez-Albalat, B. (1990): "Guerra y religión en la Gallaecia y la Lusitania Antiguas". Ediciós do Castro. Coruña.

LINCOLN, B. (1991): "Sacerdotes, guerreros y ganado. Un estudio sobre la ecologia de las religiones". Akal. Madrid.

Meiuide Camesel.le. G. (1991): "La Edad del Bronce en Galicia". "Galicia Historia", I, Prehistoria e Historia Antigua. Hércules de Ediciones. Coruña: 234-274.

Vazouez Varel.A. J. M. (1990): “Os petroglifos de Galicia". Servicio de Publicacions da Universidade de Santiago. Santiago de Compostela.

- (1991): "Ideología y poder en el arte rupestre prehistórico gallego". Cuadernos de Estudios Gallegos, XXXIX, 104: 15-22.

- (1992): "Voces del pasado: La ideología profana de la Protohistoria de Galicia a través de los petroglifos al aire libre". "Finis Terrae, Estudios en lembranza do Prof. Dr. Alberto Balil". Santiago de Compostela: 6170.

- (1993): “Arte Prehistórico". "Galicia Arte", IX, Arte Prehistórico y Romano. Hércules de Ediciones. Coruna: 17-233.

- (1994): "Ritos y creencias de la prehistoria gallega". Xuntanza Editorial. Coruña. 